\title{
Exact and approximate symmetry projectors for the electronic structure problem on a quantum computer
}

\author{
Tzu-Ching Yen, Robert A. Lang, and Artur F. Izmaylov* \\ Department of Physical and Environmental Sciences, \\ University of Toronto Scarborough, Toronto, Ontario, M1C 1A4, \\ Canada; and Chemical Physics Theory Group, Department of Chemistry, \\ University of Toronto, Toronto, Ontario, M5S 3H6, \\ Canada; email of the corresponding author: artur.izmaylov@utoronto.ca
}

(Dated: October 8, 2019)

\begin{abstract}
Solving the electronic structure problem on a universal-gate quantum computer within the variational quantum eigensolver (VQE) methodology requires constraining the search procedure to a subspace defined by relevant physical symmetries. Ignoring symmetries results in convergence to the lowest eigenstate of the Fock space for the second quantized electronic Hamiltonian. Moreover, this eigenstate can be symmetry broken due to limitations of the wavefunction ansatz. To address this VQE problem, we introduce and assess methods of exact and approximate projectors to irreducible eigen-subspaces of available physical symmetries. Feasibility of symmetry projectors in the VQE framework is discussed, and their efficiency is compared with symmetry constraint optimization procedures. Generally, projectors introduce higher numbers of terms for VQE measurement compare to the constraint approach. On the other hand, the projection formalism improves accuracy of the variational wavefunction ansatz without introducing additional unitary transformations, which is beneficial for reducing depths of quantum circuits.
\end{abstract}

\section{INTRODUCTION}

The variational quantum eigensolver (VQE) method $^{1-3}$ is one of the most practical approaches to the electronic structure problem on current and near future universal quantum computers. VQE finds the best approximation to the lowest eigenstate of a Hamiltonian in a manifold of wavefunctions by a variational energy minimization procedure involving both quantum and classical computers. The quantum computer operates in terms of qubit operators and qubit wavefunctions; its role is to set up a trial qubit wavefunction and to measure its energy on a qubit Hamiltonian for obtaining the energy expectation value. To reformulate the system electronic Hamiltonian $\left(\hat{H}_{e}\right)$ in a qubit form $\left(\hat{H}_{q}\right)$, usually, $\hat{H}_{e}$ is taken in the second quantized form and is transformed iso-spectrally using the fermion-spin transformations such as JordanWigner $(J W)^{4,5}$ or more resource-efficient Bravyi-Kitaev (BK) ${ }^{6-10}$ In this setup, a quantum hardware measures the expectation value of the energy on the current trial wavefunction. Then, a classical computer determines a new trial wavefunction based on the information collected by the quantum hardware. These two steps are iterated until convergence of the energy. The VQE was successfully employed on several quantum computers and used for few small molecules up to $\mathrm{BeH}_{2}{ }^{11}$ and $\mathrm{H}_{2} \mathrm{O} \cdot{ }^{12,13}$

Note that even though the wavefunction optimization problem is not solved on a quantum computer, the main advantage of the hybrid VQE scheme is a compact representation of a unitary ansatz for the wavefunction. One of the standard unitary hierarchies of approximations for the wavefunction is the unitary coupled cluster (UCC) method. At any finite level of excitations starting from doubles the number of terms in UCC equations grow exponentially with the size of the system on a classical com- puter, while the number of parameters for the VQE optimization grows only polynomially. It was found recently that even though the UCC hierarchy is generally more efficient than a regular coupled cluster hierarchy in capturing the electron correlation energy, it still breaks down for strongly correlated models. ${ }^{14}$

One of the VQE issues is that, in most implementations, it operates in the Fock space of the original fermionic problem, which maps iso-spectrally onto the Hilbert space of $N$ qubits. Thus, states of all possible number of electrons are present in the qubit Hilbert space. To optimize a particular electronic state with a fixed number of electrons or spin it is necessary to constrain the search process to a particular segment of the qubit Hilbert state. ${ }^{2}$ Previously, symmetry constraints were introduced via penalty functions. ${ }^{12}$ These penalties guarantee the optimal wavefunction to have the correct symmetry only in its expectation values while corresponding variances can be nonzero. Recently, solutions to this problem for some symmetries was proposed through introducing symmetry-preserving circuits. ${ }^{15,16}$ Additionally, using symmetries one can reduce the number of qubits in the Hamiltonian and thus facilitate optimization of the electronic state. ${ }^{17}$

Interestingly, symmetries in VQE are not only necessities for accessing higher states in the Fock space but also means to improve the accuracy. Some simple symmetries (e.g. the parity of the electronic number) were used to mitigate errors originating from noise by measuring entangled ancilla qubits. ${ }^{18,19}$ Also, due to commutativity of some Hamiltonian terms with the electron number symmetry operator, errors in preparation or measurement of the wavefunction can be identified from the wavefunction read-out after measurement of these terms. ${ }^{12}$

In this paper, we investigate whether projectors based 
on Hamiltonian symmetries can be a better alternative to constraints. General procedures of introducing both constraints and projectors are considered. A particular attention is dedicated to generating compact qubit forms for symmetry projectors, which was a problem discovered in earlier studies. ${ }^{20,21}$ This appears to be possible only for some symmetries while for others only approximate expressions are feasible. Generally, time complexity of a single step of VQE is tied to the number of terms to be measured and their variances. Only single qubit measurements are available in the current architectures, this limits the elementary measurable operator parts to linear combinations of operators that commute with each other at a single qubit level. ${ }^{22}$ Recently, several new techniques to reduce the number of linear combinations requiring separate measurements have been introduced. ${ }^{23-25}$

Apart from imposing physical symmetries to converge to the right state, symmetry projectors also allow more efficient search for the wavefunction of strongly correlated systems on a classical computer. ${ }^{26}$ In this context, introducing symmetry projectors into the variational procedure (i.e. variation-after-projection type of methods) provides more efficient use of variational parameters in the wavefunction ansatz. One rationale for this is that the wavefunction ansatz does not need to use variational parameters for maintaining the right symmetry but only to lower the energy. Thus, development of projection techniques in quantum computing can be an efficient approach to treatment of strongly correlated systems.

The rest of the paper is organized as follows. Section II presents theory of symmetry projection construction, compares different ways of use of projectors and constraints in the VQE procedure, discusses approaches to building approximate projectors and their relations with constraints, and provides qubit space expressions for operators involved in VQE optimization. In Sec. III we assess various symmetry projection and constraint techniques on a set of three molecular systems $\left(\mathrm{H}_{2}, \mathrm{LiH}\right.$, and $\mathrm{H}_{2} \mathrm{O}$ ) within the qubit mean-field approach. Section IV concludes by providing summary and outlook.

\section{THEORY}

\section{A. Use of symmetry}

Here we review basic elements of symmetry use in the eigenvalue problem. Most of the material can be found in various textbooks, ${ }^{27-29}$ but to keep the paper self-contained we review it here with a special emphasis on treatment of multiple symmetry operators that do not generally commute with each other (i.e. non-abelian case).

For any Hamiltonian one can find a set of operators $\left\{\hat{O}_{i}\right\}$ commuting with the Hamiltonian, $\left[\hat{H}, \hat{O}_{i}\right]=0$. However, in general, these operators do not commute with each other $\left[\hat{O}_{i}, \hat{O}_{j}\right] \neq 0$. On the one hand, noncommutativity introduces a problem that there is no com- mon set of eigenfunctions for all symmetry operators, but on the other hand, it allows us to generate additional operators that commute with $\hat{H}$ by forming all possible nontrivial commutators within the $\left\{\hat{O}_{i}\right\}$ set. This process leads to an algebraic structure, where all $\hat{O}_{i}$ operators in the augmented set satisfy the condition

$$
\left[\hat{O}_{i}, \hat{O}_{j}\right]=\sum_{k} c_{i j}^{(k)} \hat{O}_{k}
$$

here $c_{i j}^{(k)}$ are some constants. Mathematically speaking, we have obtained a Lie algebra which consists of operators commuting with the Hamiltonian and thus are referred to as symmetries.

In this general case, the eigenfunctions of the Hamiltonian correspond to particular irreducible eigen-subspaces of the symmetry operators which are not necessarily one dimensional as in the abelian case. Considering the structure of these irreducible eigen-subspaces is necessary to build proper constraints or projectors.

For the purpose of construction projectors on eigenspaces of the symmetry operators $\left\{\hat{O}_{i}\right\}$ it is useful to separate two cases based on whether in addition to the Lie algebraic structure $\left\{\hat{O}_{i}\right\}$ form a multiplicative group $\hat{O}_{i} \hat{O}_{j}=\hat{O}_{k}$ (e.g. point group symmetries) or not (e.g. electron spin $s u(2)$ Lie algebra).

Existence of the group structure allows one to generate projectors on the group irreducible representations following the standard procedure

$$
\hat{P}_{\Gamma}=\frac{d_{\Gamma}}{|G|} \sum_{k=1}^{|G|} \chi_{\Gamma}^{*}\left(\hat{O}_{k}\right) \hat{O}_{k},
$$

where $\Gamma$ is the irreducible representation of interest, $d_{\Gamma}$ is the dimension of $\Gamma$, and $\chi_{\Gamma}\left(\hat{O}_{k}\right)$ are characters for the group elements. $\hat{O}_{k}$ are generally not unitary operators, but any finite group can be represented as a set of unitary operators. Therefore, we will consider $\hat{O}_{k}$ 's forming a finite group as unitary.

In absence of a group structure, the Lie algebra can be turned into a continuous Lie group using the standard exponential mapping. Then the same standard machinery as in finite groups can be extended to continuous compact groups to formulate projectors. However, switching from the algebra to a group is not necessary to obtain the projectors onto irreducible representations of the algebra. Moreover, irreducible representations of the underlining Lie algebra are still necessary for constructing projectors onto irreducible representations of the group.

Standard techniques to build irreducible representation of simple and semisimple Lie algebras (e.g. $s u(2)$, the electron spin) are well described in various mathematical textbooks ${ }^{27,28}$ and Ref. 30. We will not detail them here but only formulate the useful result that in all semisimple Lie algebras, it is straightforward to find a set of fully commuting operators whose eigenvalues characterize and eigenfunctions span all irreducible representations. For the well-known $s u(2)$-case, the usual choice of 
these operators are $\hat{S}_{z}$ and $\hat{S}^{2}$. From the computational point of view, it is convenient to present projectors for each of the operators in the commuting set $\hat{O}_{i}$ as an operator function $\hat{P}_{j}^{(i)}=F\left(\hat{O}_{i}, o_{j}^{(i)}\right)$, where $o_{j}^{(i)}$ is the eigenvalue determining the eigen-subspace of interest. Then the total projector can be written as

$$
\hat{P}_{\Gamma}=\prod_{i} F\left(\hat{O}_{i}, o_{j}^{(i)}\right), j \in \Gamma
$$

where the eigenvalues $o_{j}^{(i)}$ should be chosen so that the projection is done on a particular irreducible subspace of the Lie algebra, $\Gamma$. All operators $\hat{O}_{i}$ in Eq. (3) commute, and therefore, their projectors can be put in any order.

\section{B. Construction of projectors for individual symmetry operators}

For practical use of Eq. (3) we summarize few approaches for constructing functional forms for individual symmetry projectors $\hat{P}_{j}^{(i)}=F\left(\hat{O}_{i}, o_{j}^{(i)}\right)$, while more detailed discussion is provided in Ref. 30. The majority of symmetry operators have discrete spectra, and the corresponding function $F$ can be constructed from some differentiable representation of the Kronecker-delta function. To see this, let us present the projector as

$$
\begin{aligned}
\hat{P}_{j}^{(i)} & =\sum_{n}\left|\phi_{n}^{(i)}\right\rangle\left\langle\phi_{n}^{(i)}\right| \delta_{n j} \\
& =\sum_{n}\left|\phi_{n}^{(i)}\right\rangle\left\langle\phi_{n}^{(i)}\left|F\left(x, o_{j}^{(i)}\right)\right|_{x=o_{n}^{(i)}},\right.
\end{aligned}
$$

where $\left|\phi_{n}^{(i)}\right\rangle$ are the eigenfunctions of $\hat{O}_{i}$ corresponding to eigenvalues $o_{n}^{(i)}$. Here, we substituted the Kroneckerdelta function $\delta_{n j}$ with a differentiable function $F\left(x, o_{j}^{(i)}\right)$ of the form

$$
F\left(x, o_{j}^{(i)}\right)=\left\{\begin{array}{l}
1, x=o_{j}^{(i)}, \\
0, x=o_{n}^{(i)}, n \neq j, \\
\xi(x) \in[0,1], x \neq o_{n}^{(i)}, \forall n,
\end{array}\right.
$$

where $\xi(x)$ can be any smooth function for intermediate values of $x$. Due to its differentiability, one can expand $F$ in the Taylor series, and this expansion defines $F\left(\hat{O}_{i}, o_{j}^{(i)}\right)$. There are multiple ways to define $F\left(x, o_{j}^{(i)}\right),{ }^{30}$ here we list the two most useful:

1) Integration over a unit circle:

$$
F\left(x, o_{j}^{(i)}\right)=\frac{1}{2 \pi} \int_{0}^{2 \pi} e^{i \phi\left(x-o_{j}^{(i)}\right)} d \phi .
$$

Here, for any $x \neq o_{j}^{(i)}$ we obtain zero. Such selectivity comes with a price of introducing the integral.

2) The Lagrange interpolation product:

$$
F\left(x, o_{j}^{(i)}\right)=\prod_{n \neq j} \frac{x-o_{n}^{(i)}}{o_{j}^{(i)}-o_{n}^{(i)}},
$$

which is less restrictive since for $x$-values in between the eigenvalues the functional value is not fixed to zero or one. The Löwdin spin projector uses Eq. (8). ${ }^{31}$

Equation (8) is especially useful to build projectors for operators with finite number of eigenvalues. Interestingly, for such operators, projectors based on Eqs. (7) and (8) are the same. This is a consequence of the CayleyHamilton theorem ${ }^{32}$ because there is only a finite number of linear independent powers for operators with finite spectra. Thus, any function of such an operator is equivalent to $M-1$ polynomial, where $M$ is the number of eigenvalues.

Another interesting connection can be found between projectors based on Eq. (7) and generalization of the group projector in Eq. (2) to a continuous group. It is straightforward to see that $g\left(\hat{O}_{i}, \phi\right)=\exp \left[i \phi \hat{O}_{i}\right]$, where $\phi \in[0,2 \pi]$ forms a continuous compact oneparametric cyclic group, $g\left(\hat{O}_{i}, \phi^{\prime}\right) g\left(\hat{O}_{i}, \phi^{\prime \prime}\right)=g\left(\hat{O}_{i}, \phi\right)$, $\phi=\bmod \left(\phi^{\prime}+\phi^{\prime \prime}, 2 \pi\right)$. All cyclic groups are abelian and have one-dimensional irreducible representations, in this case, irreducible representations are characterized by $o_{j}^{(i)}$, with characters $\exp \left[i \phi o_{j}^{(i)}\right]$. Therefore,

$$
\hat{P}_{j}^{(i)}=\frac{1}{2 \pi} \int_{0}^{2 \pi} d \phi e^{i \phi\left(\hat{O}_{i}-o_{j}^{(i)}\right)},
$$

can be interpreted both as the result from Eq. (7) and as the continuous group extension of Eq. (2).

\section{Constraining the energy minimization}

Two main approaches to impose symmetry constraints in the variational search are addition of penalty functions and projecting out irrelevant symmetries. We give a brief overview of different schemes within these two approaches. It will be assumed that some unitary parametrization is used for the wavefunction, $|\psi(\theta)\rangle$, where $\theta$ is a set of parameters. Note that the only difference from the conventional VQE scheme will be in minimization of different functionals involving symmetry operators. To evaluate these functionals in the VQE fashion, a few extra expectation values need to be measured.

Adding penalty for deviation from correct average values requires minimization of the following functional

$$
\begin{aligned}
E_{c}[|\psi(\theta)\rangle] & =\langle\psi(\theta)|\hat{H}| \psi(\theta)\rangle \\
& +\mu \sum_{i}\left(\left\langle\psi(\theta)\left|\hat{O}_{i}\right| \psi(\theta)\right\rangle-o_{j}^{(i)}\right)^{2},
\end{aligned}
$$

where $\left\{\hat{O}_{i}\right\}$ are mutually commuting symmetry operators with their eigenvalues $o_{j}^{(i)}$ defining a certain irreducible subspace. This approach has been implemented in constrained VQE (CVQE) and has advantages of simplicity (only averages of the symmetry operators are needed), the shortcomings of CVQE is that the symmetry is satisfied only on average, and it is possible that variances of $\hat{O}_{i}$ are non-zero. 
Adding penalty for deviation from the correct averages and non-zero variances extends the Lagrange functional of Eq. (10) by adding penalties for variances

$$
\begin{aligned}
\tilde{E}_{c}[|\psi(\theta)\rangle]= & E_{c}[|\psi(\theta)\rangle]+\mu \sum_{i}\left(\left\langle\psi(\theta)\left|\hat{O}_{i}^{2}\right| \psi(\theta)\right\rangle\right. \\
& \left.-\left\langle\psi(\theta)\left|\hat{O}_{i}\right| \psi(\theta)\right\rangle^{2}\right)
\end{aligned}
$$

It is straightforward to show that the variance of the operator reaches its minimum (zero) only on its eigenstates (see appendix A). Thus, for the computational price of evaluating expectation values of squares of the symmetry operators we can impose the symmetry completely. Also, minimization in Eq. (11) is equivalent to modification of the Hamiltonian as

$$
\hat{H}_{c}=\hat{H}+\mu \sum_{i}\left(\hat{O}_{i}-o_{j}^{(i)}\right)^{2}
$$

in the regular variational procedure.

One unpleasant feature of both constraint approaches is the presence of arbitrary parameter $\mu$ which is usually set to a large positive number. This arbitrariness does not affect results if the variational ansatz $|\psi(\theta)\rangle$ is flexible enough to satisfy the constraint exactly. However, if this condition is not satisfied, $\mu$ can significantly affect the final energy.

Another limitation for both approaches is that treatment of non-abelian group symmetries will require introducing the projector on irreducible subspace (Eq. (2)).

Introducing projectors to penalize undesired symmetry components addresses constraining the energy minimization for non-abelian groups. If the projector to an eigenstate of the right symmetry is available then we can modify the Hamiltonian to introduce the penalty for components of undesired symmetry

$$
\hat{H}_{\Gamma}=\hat{H}+\mu\left(\mathbb{1}-\hat{P}_{\Gamma}\right) .
$$

Here we used the idempotency condition for the projector $\left(\hat{P}_{\Gamma}^{2}=\hat{P}_{\Gamma}\right)$ so that non-negative operator $\left(\mathbb{1}-\hat{P}_{\Gamma}\right)^{2}$ is reduced to $\left(\mathbb{1}-\hat{P}_{\Gamma}\right)$. This approach is generally equivalent to imposing constraints on averages and variances, but it has an advantage to be able to address the nonabelian group symmetry cases. On the other hand, it can introduce more complex dependence on the symmetry operators from Eq. (3) for $\hat{P}_{\Gamma}$.

Projecting out undesired symmetries introduces the functional that projects out undesired symmetry components from the wavefunction

$$
\begin{aligned}
E_{\Gamma}[|\psi(\theta)\rangle] & =\frac{\left\langle\psi(\theta)\left|\hat{P}_{\Gamma}^{\dagger} \hat{H} \hat{P}_{\Gamma}\right| \psi(\theta)\right\rangle}{\left\langle\psi(\theta)\left|\hat{P}_{\Gamma}^{\dagger} \hat{P}_{\Gamma}\right| \psi(\theta)\right\rangle} \\
& =\frac{\left\langle\psi(\theta)\left|\hat{H} \hat{P}_{\Gamma}\right| \psi(\theta)\right\rangle}{\left\langle\psi(\theta)\left|\hat{P}_{\Gamma}\right| \psi(\theta)\right\rangle},
\end{aligned}
$$

where the second equality is a result of the projector's commutation with $\hat{H}$, hermiticity, and idempotency. Note that the point of view that the projector modifies the wavefunction makes Eq. (15) significantly different from the approaches based on penalties. Indeed, having the projector in the denominator is an essential feature that differentiates this expression from other forms. To see this, let us consider an alternative, where a function commuting with the Hamiltonian $\hat{\mathcal{F}}$ is introduced only to the numerator

$$
\begin{aligned}
E_{\mathcal{F}}[|\psi(\theta)\rangle] & =\left\langle\psi(\theta)\left|\hat{\mathcal{F}}^{\dagger} \hat{H} \hat{\mathcal{F}}\right| \psi(\theta)\right\rangle \\
& =\left\langle\psi(\theta)\left|\hat{H} \hat{\mathcal{F}}^{\dagger} \hat{\mathcal{F}}\right| \psi(\theta)\right\rangle \\
& =\left\langle\psi(\theta)\left|\hat{H}+\mu\left(\mathbb{1}-\hat{P}_{\Gamma}\right)\right| \psi(\theta)\right\rangle,
\end{aligned}
$$

where in the last equality we defined $\hat{\mathcal{F}}^{\dagger} \hat{\mathcal{F}}=\mathbb{1}+$ $\mu \hat{H}^{-1}\left(\mathbb{1}-\hat{P}_{\Gamma}\right)$. Thus $\mathcal{F}$ can be associated with $\hat{P}_{\Gamma}$ and variational optimization of $E_{\mathcal{F}}[|\psi(\theta)\rangle]$ is equivalent to the optimization of the $\hat{H}_{\Gamma}$ Hamiltonian in Eq. (13).

\section{Approximate projectors}

As we will see further the exact projector expressions are not always feasible for an efficient implementation. Here we present two approaches for approximating projectors.

\section{Group theoretical approximation: Forming subgroups}

To simplify general expressions for the group projection in Eq. (2) or in the analogous infinite summation over the cyclic group in Eq. (9), one can reduce the summation to that over a subgroup that permits an efficient implementation. For example, integration over an infinite cyclic group can be substituted by a summation over a finite cyclic subgroup. Note that for operators with spectra where ratios of spectral gaps for the eigenvalue with its neighbors form a finite set of rational numbers (e.g., $\hat{S}^{2}, \hat{S}_{z}$ or the number of electrons operator, $\hat{N}$ ) one can form a finite cyclic subgroup $\left\{\hat{U}^{k}\right\}$, with the generator $\hat{U}=\exp \left(2 \pi i \hat{O}_{i} /(d M)\right)$, where $d M$ is a scaling factor that makes all eigenvalues of $\hat{O}_{i}$ to be rational numbers. The characters of sought irreducible representations are $\chi_{\Gamma}\left(\hat{U}^{k}\right)=\exp (2 \pi i \Gamma k /(d M))$, where $\Gamma$ 's are eigenvalues of $\hat{O}_{i}, o_{j}^{(i)}$. The size of $\left\{\hat{U}_{k}\right\}$ can be regulated by choosing $d M$.

\section{Appoximating the Kronecker-delta function}

The exact projector operator was obtained using a form of continuous indicator function for the corresponding symmetry operator (Eq. (6)). To introduce approximations to projector operator one can be less strict on how many eigen-values will be zeroed by an approximate version of the continuous representations of the 
Kronecker-delta function

$$
\mathcal{F}\left(x, o_{j}^{(i)}\right)=\left\{\begin{array}{l}
1, x=o_{j}^{(i)}, \\
0, x=o_{n}^{(i)}, o_{n}^{(i)} \in \mathbf{S}, \\
\xi(x), x \notin \mathbf{S} \cup o_{j}^{(i)},
\end{array}\right.
$$

where $\xi(x)$ is an arbitrary function maintaining smoothness, and $\mathbf{S}$ is an incomplete set of eigen-values for the target symmetry operator that one would like to eliminate. This definition does not guarantee the idempotency when the Hermitian symmetry operator $\hat{O}_{i}$ is made an argument, $\mathcal{F}\left(\hat{O}_{i}, o_{j}^{(i)}\right)^{2} \neq \mathcal{F}\left(\hat{O}_{i}, o_{j}^{(i)}\right)$ but still preserves the Hermiticity $\mathcal{F}\left(\hat{O}_{i}, o_{j}^{(i)}\right)^{\dagger}=\mathcal{F}\left(\hat{O}_{i}, o_{j}^{(i)}\right)$. Using this approximation in the variational approach results in

$$
\begin{aligned}
E_{\mathcal{F}}[\psi] & =\frac{\left\langle\psi\left|\hat{\mathcal{F}}^{\dagger} \hat{H} \hat{\mathcal{F}}\right| \psi\right\rangle}{\left\langle\psi\left|\hat{\mathcal{F}}^{\dagger} \hat{\mathcal{F}}\right| \psi\right\rangle} \\
& =\frac{\left\langle\psi\left|\hat{H} \hat{\mathcal{F}}^{2}\right| \psi\right\rangle}{\left\langle\psi\left|\hat{\mathcal{F}}^{2}\right| \psi\right\rangle} .
\end{aligned}
$$

The wavefunction in this functional can be expanded in mutual eigenstates $\left\{\phi_{k}\right\}$ of $\hat{H}$ and symmetry operator $\hat{O}_{i}$

$$
E_{\mathcal{F}}[\psi]=\frac{\sum_{k} e_{k}\left|c_{k}\right|^{2} f_{k}^{2}}{\sum_{k}\left|c_{k}\right|^{2} f_{k}^{2}}
$$

where $|\psi\rangle=\sum_{k} c_{k}\left|\phi_{k}\right\rangle, \hat{H}\left|\phi_{k}\right\rangle=e_{k}\left|\phi_{k}\right\rangle$, and $\hat{\mathcal{F}}\left|\phi_{k}\right\rangle=$ $f_{k}\left|\phi_{k}\right\rangle . f_{k}$ is one for the target symmetry state, is zero for $\left|\phi_{k}\right\rangle$ corresponding to symmetries from the elimination set $\mathbf{S}$, and is greater than one for all other states. If the target state has the lowest energy among all states excluding those from the $\mathbf{S}$ set, the variational procedure will easily converge to the target state. Due to variational procedure, the only spurious symmetry components in $|\psi\rangle$ after the optimization of Eq. (21) can be from the $\mathbf{S}$ set. Thus, $|\psi\rangle$ can be further purified by application of the $\hat{\mathcal{F}}$ operator.

In practice, projectors satisfying these constraints can be constructed through Eq. (8)

$$
\mathcal{F}^{2}\left(x, o_{j}^{(i)}\right)=\prod_{n}^{e v e n} \frac{x-o_{n}^{(i)}}{o_{j}^{(i)}-o_{n}^{(i)}} \prod_{k}^{\text {even }} \frac{x-o_{k}^{(i)}}{o_{j}^{(i)}-o_{k}^{(i)}}
$$

where $\mathbf{S}$ consists of even numbers of $o_{k}^{(i)}$ and $o_{n}^{(i)}$ such that $o_{k}^{(i)}<o_{j}^{(i)}<o_{n}^{(i)}$. To minimize the number of terms in Eq. (23) one can take zero number of terms for one of the sets $\left(o_{k}^{(i)}\right.$ or $\left.o_{n}^{(i)}\right)$ and two for the other.

\section{E. Operators in the qubit space}

Here we summarize the Hamiltonian and all of its symmetry operators for molecules in the qubit space. For all operators, their fermionic second-quantized form, JWand BK-transformed qubit forms, exact and approximate projectors are discussed.

\section{Hamiltonian}

In order to formulate the electronic structure problem for a quantum computer that operates with qubits (twolevel systems), the electronic Hamiltonian needs to be transformed iso-spectrally to its qubit form. This is done in two steps. First, the second quantized form of $\hat{H}_{e}$ is obtained

$$
\hat{H}_{e}=\sum_{p q} h_{p q} \hat{a}_{p}^{\dagger} \hat{a}_{q}+\frac{1}{2} \sum_{p q r s} g_{p q r s} \hat{a}_{p}^{\dagger} \hat{a}_{q}^{\dagger} \hat{a}_{s} \hat{a}_{r}
$$

where $\hat{a}_{p}^{\dagger}\left(\hat{a}_{p}\right)$ are fermionic creation (annihilation) operators, $h_{p q}$ and $g_{p q r s}$ are one- and two-electron integrals in a spin-orbital basis. ${ }^{33}$ This step has polynomial complexity and is carried out on a classical computer. Then, using the $\mathrm{JW}^{4,5}$ or more resource-efficient BK transformation, ${ }^{6-10}$ the electronic Hamiltonian is converted isospectrally to a qubit form

$$
\hat{H}_{q}=\sum_{I} C_{I} \hat{W}_{I}
$$

where $C_{I}$ are numerical coefficients, and $\hat{W}_{I}$ are Pauli "words", products of Pauli operators of different qubits

$$
\hat{W}_{I}=\cdots \hat{\sigma}_{2}^{(I)} \hat{\sigma}_{1}^{(I)},
$$

$\hat{\sigma}_{i}^{(I)}$ is one of the $\hat{x}, \hat{y}, \hat{z}$ Pauli operators for the $i^{\text {th }}$ qubit. The number of qubits $N$ is equal to the number of spinorbitals used in the second quantized form [Eq. (24)]. Since every fermionic operator is substituted by a product of Pauli operators in both JW and BK transformations, the total number of Pauli words in $\hat{H}_{q}$ scales as $N^{4}$.

\section{Electron number operator}

The electron number operator has the following forms in various representations

$$
\begin{aligned}
\hat{N} & =\sum_{p=1}^{N_{o}} a_{p}^{\dagger} a_{p}, \\
\hat{N}_{\mathrm{JW}} & =\frac{N_{q}}{2}-\frac{1}{2} \sum_{k=1}^{N_{q}} \hat{z}_{k}, \\
\hat{N}_{\mathrm{BK}} & =\frac{N_{q}}{2}-\frac{1}{2} \sum_{k=1}^{N_{q}} \underline{\hat{z}_{F(k)}},
\end{aligned}
$$

where $N_{o}$ is the number of orbitals $\left(N_{o}=N_{q}\right), F(k)$ is the flip set of qubit $k$, and $F(k)=F(k) \cup k,{ }^{7}$ while $\hat{z}_{F(k)}$ stands for $\hat{z}$ 's applied to all qubits in $F(k)$. Let us consider the exponential form of the projector to the 
number of electrons

$$
\begin{aligned}
\hat{P}_{N}= & \frac{1}{2 \pi} \int_{0}^{2 \pi} d \phi e^{i \phi(\hat{N}-N)} \\
= & \frac{1}{2 \pi} \int_{0}^{2 \pi} d \phi e^{-i \phi N} \prod_{k=1}^{N_{q}} e^{i \phi\left(c_{k} \hat{W}_{k}\right)} \\
= & \frac{1}{2 \pi} \int_{0}^{2 \pi} d \phi e^{-i \phi N} \prod_{k=1}^{N_{q}}\left[\cos \left(c_{k} \phi\right) \mathbb{1}\right. \\
& \left.+i \hat{W}_{k} \sin \left(c_{k} \phi\right)\right],
\end{aligned}
$$

where $\hat{W}_{k}$ are Pauli words constituting $\hat{N}$ and $c_{k}$ are corresponding coefficients, all $\hat{W}_{k}$ are mutually commuting therefore exponent of $\hat{N}$ is presented as a product of $c_{k} \hat{W}_{k}$ exponents. The last integral in Eq. (32) contains $2^{N_{q}}$ terms, hence the scaling of the number of terms in this projector is exponential with $N_{q}$. The origin of this problem can be traced to ultra-high precision of the projector in Eq. (30), it separates an $N$-electron component from any other component even if the number of electrons in the separated components are different from the desired number by infinitesimal amount (e.g. $N \pm 10^{-10}$ ). Clearly, such precision is somewhat excessive, and if we construct a finite subgroup built of two elements $\{1, \exp [i \pi \hat{N}]\}$, projectors on irreducible representations of this cyclic subgroup can separate even/oddelectron subspaces. The important question is whether such reduction would simplify the form of the projector operator? It does for the BK and Parity forms of the $\hat{N}$ (Eq. (29))

$$
\begin{aligned}
\hat{P}_{e / o} & =\frac{1}{2}\left\{\mathbb{1} \pm \exp \left[i \pi \hat{N}_{\mathrm{BK}}\right]\right\} \\
& =\frac{\mathbb{1} \pm \hat{z}_{N_{q}}}{2} .
\end{aligned}
$$

This is a well-known symmetry in the BK or parity transformations, where the last qubit encodes information of the parity of the number of electrons.

\section{Electron spin operators}

Using the second-quantized expressions for $S_{z}$ and its BK and JW transformations one can write

$$
\begin{aligned}
\hat{S}_{z} & =\frac{1}{2} \sum_{p=1}^{N_{o} / 2} a_{p \alpha}^{\dagger} a_{p \alpha}-a_{p \beta}^{\dagger} a_{p \beta} \\
\hat{S}_{z, \mathrm{JW}} & =\frac{1}{4} \sum_{p=1}^{N_{q} / 2}-\hat{z}_{p \alpha}+\hat{z}_{p \beta} \\
\hat{S}_{z, \mathrm{BK}} & =\frac{1}{4} \sum_{k=1}^{N_{q} / 2}-\underline{\hat{z}_{F(2 k-1)}}+\underline{\hat{z}_{F(2 k)}}
\end{aligned}
$$

Similarly for the $\hat{S}_{+}$component of $\hat{S}^{2}=\hat{S}_{+} \hat{S}_{-}+\hat{S}_{z}^{2}+\hat{S}_{z}$ we can write

$$
\begin{aligned}
\hat{S}_{+} & =\sum_{p=1}^{N_{o} / 2} a_{p \alpha}^{\dagger} a_{p \beta} \\
\hat{S}_{+, \mathrm{JW}} & =\frac{1}{4} \sum_{k=1}^{N_{q} / 2}\left(\hat{x}_{2 k-1}-i \hat{y}_{2 k-1}\right)\left(\hat{x}_{2 k}+i \hat{y}_{2 k}\right) \\
\hat{S}_{+, \mathrm{BK}} & =\frac{1}{4} \sum_{k=1}^{N_{q} / 2}\left(1-z_{\underline{F(2 k)} \backslash 2 k-1}\right)\left(\hat{x}_{2 k-1}-i \hat{y}_{2 k-1}\right)
\end{aligned}
$$

while $\hat{S}_{-}=\hat{S}_{+}^{\dagger}$ in all forms. Equations (37), (39) and (40) assume the spin-orbital's ordering $(\alpha, \beta, \alpha, \beta \ldots)$. If we use the exponential function to build projectors for $\hat{S}_{z}$ and $\hat{S}^{2}$ the same problems as in the case of $\hat{N}$ will appear. Moreover, in the $\hat{S}^{2}$ case, the exponent contains non-commuting Pauli words, which complicates obtaining final expression as a product of exponents of Pauli words even further. On the other hand, projector built from $\hat{S}_{z}$ has limited use. It can be used to avoid singlet solution through projecting out $S_{z}=0$ but it cannot guarantee singlet solution through projecting out all $S_{z} \neq 0$.

It is more natural to build approximations for projectors of these spin operators based on the Löwdin projection based on Eq. (8)

$$
\hat{P}_{S}=\prod_{S_{j} \neq S} \frac{\hat{S}^{2}-S_{j}\left(S_{j}+1\right)}{S(S+1)-S_{j}\left(S_{j}+1\right)} .
$$

contains potentially a large number of powers of the $\hat{S}^{2}$ operator, which increases the computational cost of $P_{S}$. To build approximate functions similar to the discussed $\mathcal{F}$ in Eq. (21) we suggest to resort to products limited in $S$. The spin eigenvalues included in the product correspond to the $\mathbf{S}$ subset of Eq. (19) and their eigenstates are projected out exactly. It is assumed that eigenstates that are not projected out are higher in energy and the variational procedure will avoid them. To minimize powers of $\hat{S}^{2}$ one can approximate $\hat{\mathcal{F}}^{2}$ directly using a limited product with an additional requirement of non-negativity. Also, it can be assumed that the projection on even and odd number of electrons can always be done easily. For example, to construct an approximate non-negative singlet projector within the even number of electrons subspace that will project out triplet states one can use

$$
\hat{\mathcal{F}}_{S=0}^{2}=\left(\frac{2-\hat{S}^{2}}{2}\right)\left(\frac{6-\hat{S}^{2}}{6}\right),
$$

this projector also eliminates quintet states.

\section{Point group symmetry operators}

Assuming $\left\{\hat{O}_{k}\right\}$ are elements of a finite point group $G$, their reducible matrix representations $\mathbf{O}^{(k)}$ in a given 
set of symmetry adapted orbitals $\left\{\phi_{1}, \phi_{2}, \ldots, \phi_{N_{o}}\right\}$ have elements $O_{i j}^{(k)}=\left\langle\phi_{i}\left|\hat{O}_{k}\right| \phi_{j}\right\rangle$ and possess block diagonal forms. Dimensionalities of non-zero blocks are determined by dimensionalities of the corresponding irreducible representations (e.g., $A, B, E, T$, etc). All one-dimensional irreducible representations are given by $O_{i i}^{(k)}=\chi_{\Gamma, i}\left(\hat{O}_{k}\right) \in\{-1,1\}$, where $\chi_{\Gamma, i}\left(\hat{O}_{k}\right)$ is the character for the irreducible representation $\Gamma$ of the $i^{\text {th }}$ orbital under action of $\hat{O}_{k}$.

In second quantization, the unitary orbital transformation corresponding to $\hat{O}_{k}$ is

$$
\hat{O}_{k}=\exp (-\hat{\kappa}), \quad \hat{\kappa}=\sum_{i j} \kappa_{i j} a_{i}^{\dagger} a_{j}
$$

where $\kappa_{i j}$ are elements of anti-Hermitian block-diagonal matrix $\boldsymbol{\kappa}=-\ln \left(\mathbf{O}^{(k)}\right)$. Both $\hat{\kappa}$ and $\boldsymbol{\kappa}$ differ for different group elements $\hat{O}_{k}$ and have a dependence on $k$, but for notational simplicity we keep this dependence implicit.

For one-dimensional irreducible representations $\kappa_{i i} \in$ $\{0, i \pi\}$. Hence, for the abelian groups, where all irreducible representations are one-dimensional, the second quantized orbital transformation operator $\hat{O}_{k}$ is

$$
\hat{O}_{k}=\prod_{j} \exp \left(-\kappa_{j j} a_{j}^{\dagger} a_{j}\right)
$$

This results in the following forms of the JW, parity, and BK representations

$$
\begin{aligned}
\hat{O}_{k, J W} & =\prod_{i^{*}} \hat{z}_{i^{*}} \\
\hat{O}_{k, P} & =\prod_{i^{*}} \hat{z}_{i^{*}} \hat{z}_{i^{*}-1} \\
\hat{O}_{k, B K} & =\prod_{i^{*}} \underline{\hat{z}_{F\left(i^{*}\right)}},
\end{aligned}
$$

where $\left\{\phi_{i^{*}}\right\}$ is a subset of the orbitals such that $\chi_{\Gamma\left(i^{*}\right)}=$ $-1 \forall \phi_{i^{*}}$.

For a general case of a non-abelian group some orbitals can correspond to irreducible representations of non-unit dimensionality. Due to anti-hermiticity, $\hat{\kappa}$ can be recast as

$$
\begin{aligned}
\hat{\kappa}= & \sum_{i} \kappa_{i i} a_{i}^{\dagger} a_{i} \\
& -\sum_{i<j}\left(\Re\left(\kappa_{i j}\right)\left(a_{j}^{\dagger} a_{i}-a_{i}^{\dagger} a_{j}\right)-i \Im\left(\kappa_{i j}\right)\left(a_{i}^{\dagger} a_{j}+a_{j}^{\dagger} a_{i}\right)\right),
\end{aligned}
$$

from which the qubit-space operator may be obtained by fermion-to-qubit mappings for the JW and party representations

$$
\begin{aligned}
\hat{\kappa}_{J W} & =\sum_{i} \frac{\kappa_{i i}}{2}\left(\mathbb{1}-\hat{z}_{i}\right)-\frac{i}{2} \sum_{i<j}\left(\Re\left(\kappa_{i j}\right)\left(\hat{y}_{i} \hat{x}_{j}-\hat{x}_{i} \hat{y}_{j}\right)-\Im\left(\kappa_{i j}\right)\left(\hat{y}_{i} \hat{y}_{j}+\hat{x}_{i} \hat{x}_{j}\right)\right) \hat{z}_{i \leftrightarrow j} \\
\hat{\kappa}_{P} & =\sum_{i} \frac{\kappa_{i i}}{2}\left(\mathbb{1}-\hat{z}_{i} \hat{z}_{i-1}\right)-\frac{i}{2} \sum_{i<j}\left(\Re\left(\kappa_{i j}\right)\left(\hat{z}_{i-1} \hat{x}_{i} \hat{y}_{j-1}-\hat{y}_{i} \hat{x}_{j-1} \hat{z}_{j}\right)+\Im\left(\kappa_{i j}\right)\left(\hat{y}_{i} \hat{y}_{j-1}+\hat{z}_{i-1} \hat{x}_{i} \hat{x}_{j-1} \hat{z}_{j}\right)\right) \hat{x}_{i \leftrightarrow j-1},
\end{aligned}
$$

where $\hat{\sigma}_{i \leftrightarrow j}(\sigma=x, y, z)$ denotes products $\hat{\sigma}_{i+1} \ldots \hat{\sigma}_{j-1}$. The BK transformed expression has a similar structure but in its general form is more complicated to write.

Exponentiation of the off-diagonal elements gives a linear combination of Pauli words with an upper bound of $2^{2 d-1}$ terms per a $d$-dimensional block in $\hat{\kappa}$. Individual blocks commute, thus the total complexity of implementing $\hat{O}_{k}$ has upper bound $\prod_{i}^{M} 2^{2 d_{i}-1}$ for $M d_{i}$-dimensional blocks. In practice, these estimates are too conservative because there are some cancellations that require knowledge of a particular algebra. We illustrate the full process of constructing the projectors for all irreducible representations of the $C_{3 v}$ group for a doubly degenerate $E$-type orbital basis in appendix B.

When constructing finite point group projectors, one can tailor the level of symmetry employed, depending on the subspace of interest. For example, given two irreducible representations $\Gamma$ and $\Gamma^{\prime}$ of group $G$ such that projection by $P_{\Gamma}$ and $P_{\Gamma^{\prime}}$ yield two distinct subspaces, one may consider building the projectors in a proper subgroup $H<G$, under condition that $\Gamma$ and $\Gamma^{\prime}$ remain distinct through the descent in symmetry $H \leftarrow G$. This has the practical advantage of reducing the number of unitary operations in $P_{\Gamma}$ and $P_{\Gamma^{\prime}}$ since $|H|<|G|$. Furthermore, for high symmetry molecular systems such as those belonging to linear groups $D_{\infty h}$ and $C_{\infty v}$, construction of the projectors in an overgroup $F>G$ may further split the spectrum, such that $G$ is a proper subgroup of the highest-order group the polyatomic system of interest belongs to (e.g. $D_{\infty h}$ or $C_{\infty v}$ ), under condition that 
irreducible representation $\Gamma$ of group $G$ may split to irreducible representations $\Gamma^{\prime}, \Gamma^{\prime \prime}, \ldots$ by ascent in symmetry $G \rightarrow F$. Thus, the full set of available symmetry elements to the molecular system may be viewed as a practical resource in the context of point group projectors, for the extent of which we employ is available as choice.

\section{Low-qubit-number symmetries}

One of the difficulties in implementing projectors of regular symmetries (e.g., number of electrons and spin) stems from involvement of all qubits in their operators. Here, we suggest that in some cases, it is possible to obtain few-qubit operators that commute with the molecular Hamiltonian. Finding such symmetry operators can be done by considering zero commutator problem $\left[\hat{H}, \hat{O}\left(\alpha_{i}\right)\right]=0$, where $\alpha_{i}$ are $\hat{O}$ 's parameters as a linear algebra problem in the space of Pauli words. For example, any single qubit operator can be parametrized as $\alpha_{1} \hat{x}+\alpha_{2} \hat{y}+\alpha_{3} \hat{z}$, its commutator equation with the Hamiltonian will have $\alpha_{1}=\alpha_{2}=0, \alpha_{3}=1$ solution for the last qubit in the BK transformed Hamiltonian. Similarly, one can do excessive search with two-qubit operators where the total number of parameters is 15 for each pair. Building a projector on eigen-spaces of such few-qubit operators can be done using the exponentiation (Eq. (9)).

\section{NUMERICAL RESULTS AND DISCUSSION}

To assess developed projector expressions we apply them in evaluation of potential energy surfaces (PESs) for the $\mathrm{H}_{2}$ and $\mathrm{LiH}$ molecules within the STO-3G basis and for the $\mathrm{H}_{2} \mathrm{O}$ molecule within the 6-31G basis. To generate qubit Hamiltonians, the BK transformation was used for $\mathrm{H}_{2}$ and $\mathrm{H}_{2} \mathrm{O}$ while the parity transformation was employed for $\mathrm{LiH}$. For each system two qubits are stationary (2nd and 4th in $\mathrm{H}_{2}$, 3rd and 6th in $\mathrm{LiH}$, and 4th and 8th in $\left.\mathrm{H}_{2} \mathrm{O}\right) .{ }^{34}$ Therefore, the $\hat{z}$ operators for these qubits were substituted with eigenvalues \pm 1 so that solutions of interest are within the reduced subspace. This reduction is equivalent to projecting to the even number of electrons using Eq. (34), and thus, the $\hat{P}_{e / o}$ projector is not going to be used further.

We used the qubit mean-field (QMF) ${ }^{35}$ and qubit coupled cluster $(\mathrm{QCC})^{34}$ wavefunction ansätze for all calculations with the exception of exact energies, which were evaluated via full diagonalization of the qubit Hamiltonians. The QMF wavefunction is a product of single-qubit coherent states,

$$
\begin{aligned}
|\boldsymbol{\Omega}\rangle & =\prod_{i=1}^{N_{q}}\left|\Omega_{i}\right\rangle, \\
\left|\Omega_{i}\right\rangle & =\cos \left(\frac{\theta_{i}}{2}\right)|0\rangle+\sin \left(\frac{\theta_{i}}{2}\right) e^{i \phi_{i}}|1\rangle,
\end{aligned}
$$

where $N_{q}$ is the number of qubits, $\boldsymbol{\theta}=\left\{\theta_{1}, \ldots, \theta_{N}\right\}$ and $\phi=\left\{\phi_{1}, \ldots, \phi_{N}\right\}$ are the corresponding Bloch angles taken as variational parameters. The QCC wavefunction takes the form

$$
|\Psi\rangle=\prod_{k=1}^{N_{E}} e^{i \tau_{k} \hat{W}_{k}}|\boldsymbol{\Omega}\rangle
$$

where $\tau_{k}$ are real-valued amplitudes as additional variational parameters, and $\hat{W}_{k}$ are the Pauli words [Eq. (26)] chosen using the energy gradient criterion described in Ref. 34.

For the projector formalism, the energies are evaluated through Eqs. (15) and (21) for exact and approximate projectors respectively. Table I illustrates the increase in the number of Pauli words from introducing projector operators. To compare the projector formalism with its constraining alternative, we provide results of constrained QMF (CQMF) and QCC (CQCC) calculations where both averages and variances of symmetry operators were constrained [Eq. (11)].

a) Number of electrons: The qubit reduction restricted the number of electrons in the three molecules to the following sets: $(0, \mathbf{2}, 4)$ for $\mathrm{H}_{2},(0, \mathbf{2}, 4,6)$ for $\mathrm{LiH}$, and $(0,2,4,6,8)$ for $\mathrm{H}_{2} \mathrm{O}$, where we highlight in bold the neutral configurations. Based on these configurations it is clear that approximation for the electron number projector $\mathcal{F}_{N}^{2}$ as in Eq. (23) is only possible for $\mathrm{LiH}$ and $\mathrm{H}_{2} \mathrm{O}$ by projecting $(4,6)$ and $(6,8)$ subspaces respectively. For $\mathrm{H}_{2}$, the only electron number projector is the exact one, $\hat{P}_{N}$, which projects $(0,4)$ subspaces. Variation-afterprojection (VAP) with $\hat{P}_{N}$ for $\mathrm{H}_{2}$ recovers the ground state obtained through full diagonalization of the qubit Hamiltonian (Fig. 1). Similarly for $\mathrm{LiH}, \hat{P}_{N}$ in VAP achieves significant energy lowering compared to the constrained counterpart. In addition, we found that using approximate projector $\mathcal{F}_{N}^{2}$ results in an identical curve to that of $\hat{P}_{N}$ (Fig. 2). The "hump" on the CQMF $(N=2)$ curve is associated with the spin symmetry breaking between singlet and triplet configurations. For $\mathrm{H}_{2} \mathrm{O}, \hat{P}_{N}$ and $\mathcal{F}_{N}^{2}$ produce insignificant energy lowerings for $R \leq 1.75 \AA$, for larger bond distances QMF solutions switch to $S^{2}=6$ and exact and approximate projectors do not affect energy values (Fig. 3).

For all three systems projectors result in no more than twice of the number of terms of the original Hamiltonian. Interestingly, due to some term cancellation, the approximate projector generated more terms than the exact one in its product with the $\mathrm{H}_{2} \mathrm{O}$ Hamiltonian (see Table I).

b) Electron spin: After the qubit reduction, the $\mathrm{H}_{2}$ Hamiltonian is in the singlet subspace and therefore does not require any spin projection, the $\mathrm{LiH}$ Hamiltonian has only singlet and triplet subspaces, and the $\mathrm{H}_{2} \mathrm{O}$ Hamiltonian contains singlet, triplet, and quintet subspaces. These restrictions make approximate singlet spin projections equal to the exact one for $\mathrm{LiH}$ and $\mathrm{H}_{2} \mathrm{O}$. For both systems, the QMF state undergoes symmetry-breaking transition as internuclear distance increases. Using the 


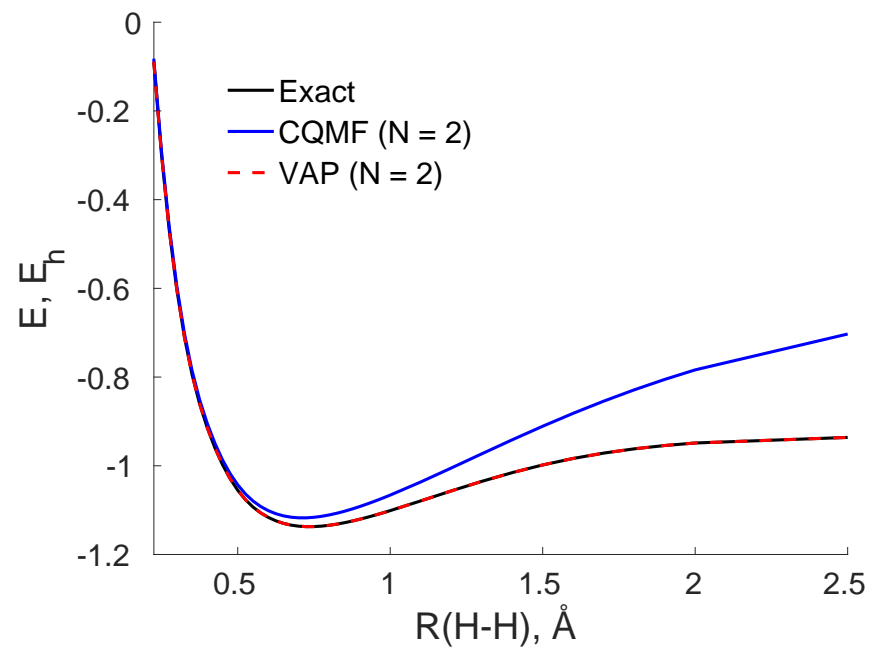

FIG. 1. PESs of $\mathrm{H}_{2}$ evaluated in the neutral $(N=2)$ subspace.

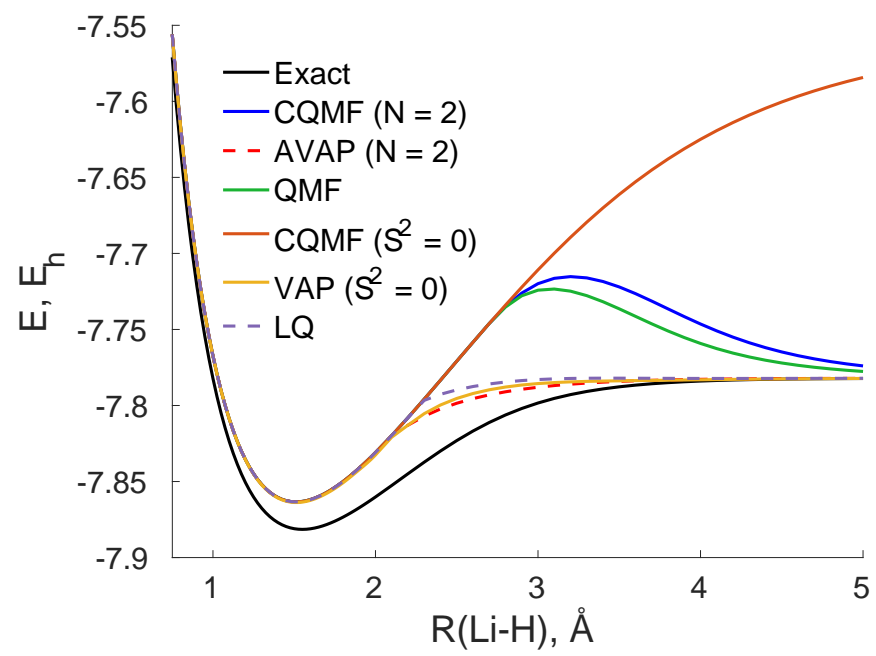

FIG. 2. PESs of LiH evaluated in variety of subspaces: neutral $(N=2)$, singlet $\left(S^{2}=0\right)$, and the low-qubit-number (LQ) symmetry with $N+2 S_{z}=2\left(S_{z}=0\right)$.

singlet projectors, we obtain singlet neutral solutions for $\mathrm{LiH}$ and $\mathrm{H}_{2} \mathrm{O}$ of lower energies in comparison to CQMF (Figs. 2 and 5). However, for $\mathrm{H}_{2} \mathrm{O}$, the QMF quintet $\left(S^{2}=6\right)$ solutions at $R \geq 1.85 \AA$ are closer to the exact ground state energy than the symmetry projected singlet solutions, which indicates need for correlation for further improvement of energy of the singlet state. The low energy of the quintet is not accidental because for this high spin configuration within the considered qubit space, QMF provides the exact answer.

To assess the performance of the singlet projector in correlated methods we employ the QCC wavefunction ansatz. For $\mathrm{LiH}$, to reach $\leq 1 \mathrm{kcal} / \mathrm{mol}$ deviation from the exact result for the whole potential energy curve the QCC approach required 7 entanglers. ${ }^{34}$ With the singlet projector, use of only one entangler in QCC, $\hat{x}_{2} \hat{x}_{1} \hat{y}_{0}$, achieves the same accuracy (Fig. 4). For $\mathrm{H}_{2} \mathrm{O}$, we employed 5 entanglers with highest gradients found in

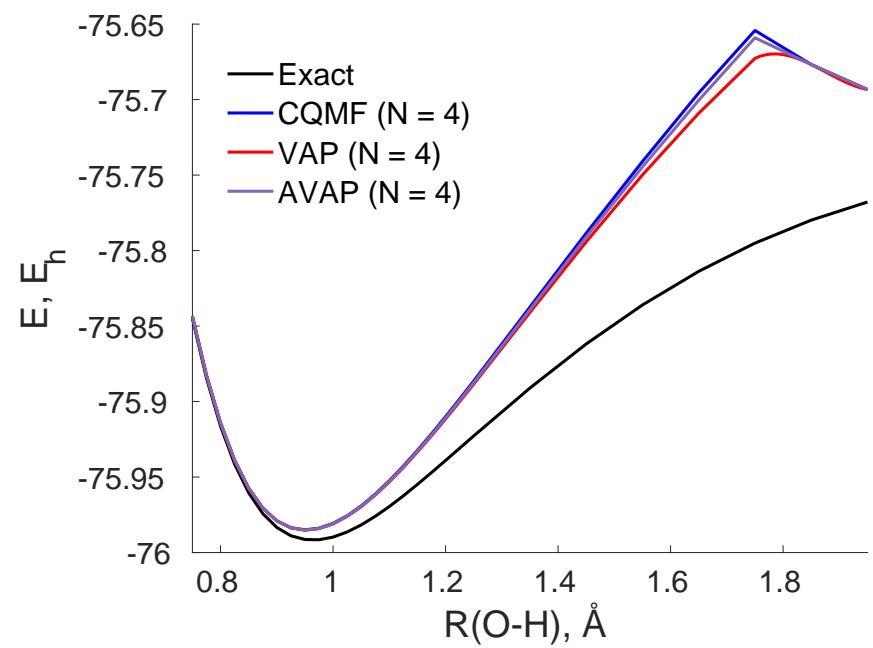

FIG. 3. PESs of $\mathrm{H}_{2} \mathrm{O}$ evaluated in the neutral $(N=4)$ subspace.

TABLE I. The number of Pauli words with coefficients $>10^{-9}$ in magnitude for various qubit space operators. Subscripts of $\hat{P}$ and $\hat{\mathcal{F}}^{2}$ denote targeted symmetries, where $L Q$ refers to the low-qubit-number symmetries, and $N$ indicates neutral species. In rows of two operators the number of unique words is shown. The Hamiltonians are taken at $R=1 \AA$, $R=3.2 \AA$ and $R=1.95 \AA$ for $\mathrm{H}_{2}, \mathrm{LiH}$ and $\mathrm{H}_{2} \mathrm{O}$, respectively.

\begin{tabular}{llll}
\hline Operators & $\mathrm{H}_{2}$ & $\mathrm{LiH}$ & $\mathrm{H}_{2} \mathrm{O}$ \\
\hline$\hat{H}$ & 6 & 100 & 165 \\
$\hat{N}$ & 3 & 7 & 9 \\
$\hat{S}^{2}$ & 0 & 40 & 71 \\
$\hat{P}_{N}$ & 2 & 16 & 32 \\
$\hat{H} \hat{P}_{N}, \hat{P}_{N}$ & 6 & 100 & 264 \\
$\mathcal{F}_{N}^{2}$ & - & 16 & 31 \\
$\hat{H} \mathcal{F}_{N}^{2}, \mathcal{F}_{N}^{2}$ & - & 100 & 320 \\
$\hat{H} \hat{P}_{S^{2}=0}, \hat{P}_{S^{2}=0}$ & - & 136 & 864 \\
$\hat{H} \hat{P}_{L Q}, \hat{P}_{L Q}$ & - & 100 & 219 \\
$\hat{P}_{B_{1}}$ & - & - & 2 \\
$\hat{H} \hat{P}_{B_{1}} \hat{H}_{\hat{H}} \hat{P}_{B_{1}} \hat{P}_{S^{2}=2}, \hat{P}_{B_{1}} \hat{P}_{S^{2}=2}$ & - & - & 242 \\
\hline
\end{tabular}

Ref. 34: $\hat{x}_{5} \hat{x}_{4} \hat{x}_{3} \hat{x}_{2} \hat{x}_{1} \hat{y}_{0}, \hat{x}_{4} \hat{x}_{3} \hat{x}_{1} \hat{y}_{0}, \hat{y}_{2} \hat{x}_{0}, \hat{x}_{4} \hat{y}_{1}$, and $\hat{x}_{3} \hat{y}_{5}$ Both QCC and QCCVAP curves achieve $\leq 1 \mathrm{kcal} / \mathrm{mol}$ deviation from the exact result near the equilibrium geometry, but away from the equilibrium, QCCVAP solutions increasingly outperform CQCC solutions (Fig. 5).

c) Low-qubit-number symmetries: For $\mathrm{LiH}$ and $\mathrm{H}_{2} \mathrm{O}$, operators involving a half of all qubits are found as the following linear combination $\hat{O}=\hat{N}+2 \hat{S}_{z}$. Projectors targeting the $\hat{O}$ subspace corresponding to neutral closedshell $\left(S_{z}=0\right)$ species were constructed. For $\mathrm{LiH}$, the projector achieves significant energy lowering with respect to the QMF solution (Fig. 2). In the $\mathrm{H}_{2} \mathrm{O}$ case, minor advantage of the $\hat{O}$ projector is present before symmetry breaking takes place in the QMF solution (Fig. 5). As expected, projectors built from symmetry involving less qubits result in the least overhead of all symmetries con- 


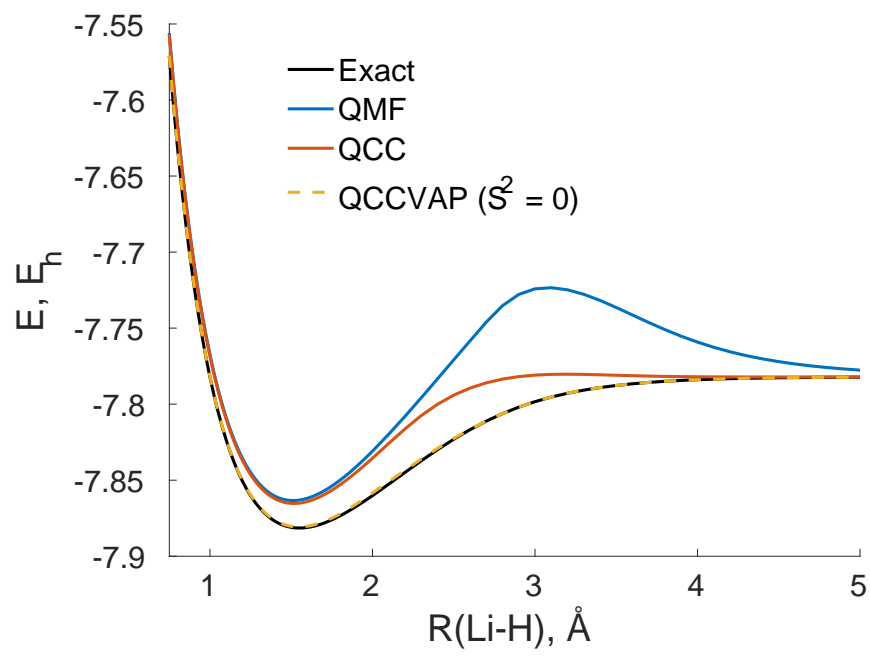

FIG. 4. PESs of LiH evaluated using both QMF and QCC wavefunction ansätze. Both QCC and QCCVAP methods use only the $\hat{x}_{2} \hat{x}_{1} \hat{y}_{0}$ entangler.

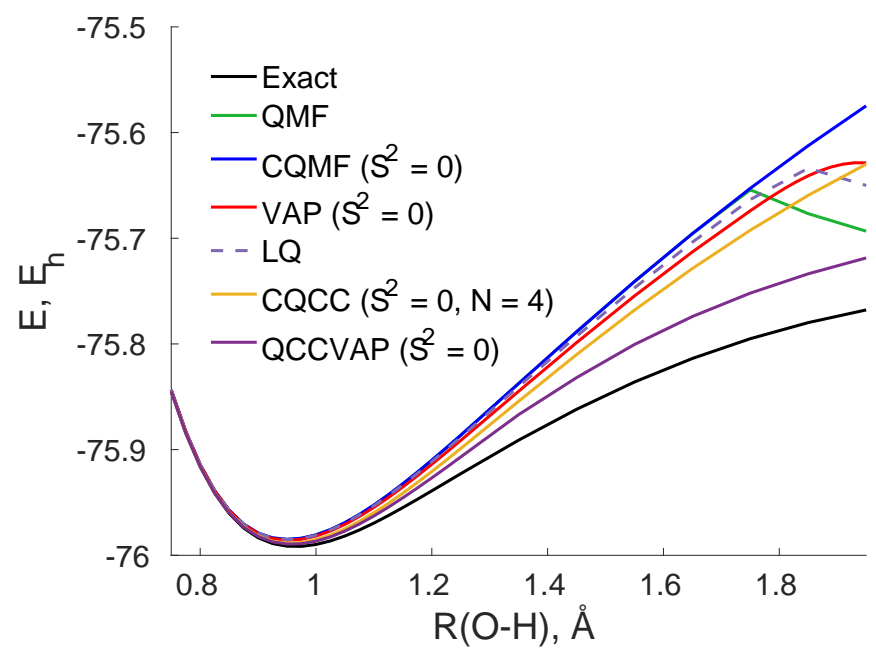

FIG. 5. PESs of $\mathrm{H}_{2} \mathrm{O}$ evaluated in the singlet $\left(S^{2}=0\right)$ subspace and the low-qubit-number (LQ) symmetry subspace with $N+2 S_{z}=4\left(S_{z}=0\right)$. Both QCC solutions use 5 entanglers $\left(\hat{x}_{5} \hat{x}_{4} \hat{x}_{3} \hat{x}_{2} \hat{x}_{1} \hat{y}_{0}, \hat{x}_{4} \hat{x}_{3} \hat{x}_{1} \hat{y}_{0}, \hat{y}_{2} \hat{x}_{0}, \hat{x}_{4} \hat{y}_{1}\right.$, and $\left.\hat{x}_{3} \hat{y}_{5}\right)$.

sidered (Table. I).

d) Point group symmetry: The reduced qubit Hamiltonians of $\mathrm{H}_{2}$ and $\mathrm{LiH}$ are entirely in single irreducible representation of the corresponding maximally abelian $D_{2 h}$ and $C_{2 v}$ groups and thus cannot benefit from projection. On the other hand, the $\mathrm{H}_{2} \mathrm{O}$ reduced Hamiltonian contains two irreducible representations of the $C_{2 v}$ group: $A_{1}$ and $B_{1}$. The projector on the $A_{1}$ irreducible subspace does not add anything to what was obtained using the number of electrons and spin projectors, therefore we illustrate capabilities of the projection onto the $B_{1}$ irreducible subspace. The lowest $B_{1}$ state in the exact solution has also triplet spin symmetry and contains 4 electrons. If we impose all three symmetry constraints in CQMF, the method cannot converge to a solution. This reveals limitations of the QMF wavefunction ansatz that cannot satisfy all constraints. Only two out of three symmetries can be satisfied in CQMF (Fig. 6). In contrast, the VAP approach can satisfy all symmetry constraints because introducing the $B_{1}$ projection does not require the QMF ansatz to satisfy the point group symmetry. Moreover, following this basic idea, introducing more than one projector $\left(B_{1}\right.$ and $\left.S^{2}\right)$ lowers the VAP energy even more.

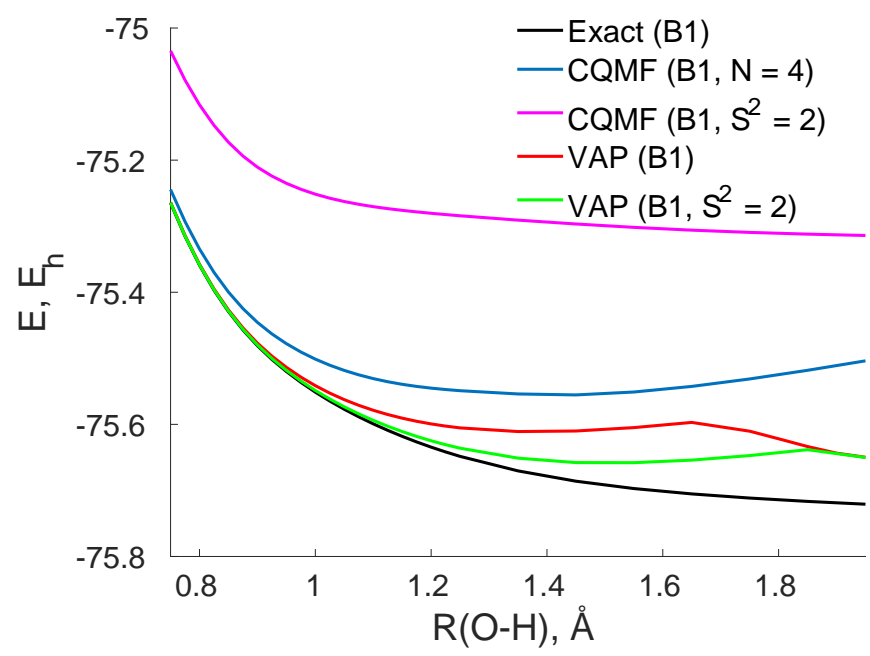

FIG. 6. PESs of $\mathrm{H}_{2} \mathrm{O}$ evaluated in the $B_{1}$ subspace. CQMF constrained to the neutral subspace $(N=4)$ has $S^{2}=1$ whereas CQMF constrained to the triplet $\left(S^{2}=2\right)$ subspace has $N=6$. Both solutions of VAP are exactly triplet and neutral on average.

\section{CONCLUSIONS}

We have considered projectors to irreducible subspaces of symmetry operators as alternatives to symmetry constraints in the variational quantum eigensolver approach to the electronic structure problem. Generally, the projector formalism generates a larger number of terms to consider than the constraint approach and thus is computationally more expensive. In cases of continuous symmetries (e.g. number of electrons and electron spin) the exact projection can involve exponentially large number of terms and therefore is infeasible without introducing approximations. Two main approaches to generating such approximations have been discussed. A higher number of terms for the projection formalism can be intuitively understood considering that the number of terms appearing from the multiplication of the Hamiltonian with the symmetry projection is usually larger than that from the addition of the constraint to the Hamiltonian.

The main advantage of using projectors is imposing symmetries without taking resources from the variational wavefunction ansatz. This helps to resolve the symmetry dilemma of variational ansätze : either to lower the energy and break symmetry or to preserve the symmetry but with higher energy. In quantum computing, use of projectors can help to reduce the depth of the wavefunc- 
tion generating circuits by shifting the computational burden on the measurement of larger number of terms appearing from projection. Additionally, the constraint and projection techniques are not mutually excluding and can be used together balancing the number of operator terms by placing some symmetries as constraints and oth- ers as projectors.

\section{ACKNOWLEDGEMENT}

A.F.I. is grateful to I. G. Ryabinkin for useful discussions. A.F.I. acknowledges financial support from the Natural Sciences and Engineering Research Council of Canada and the Ontario Early Researcher Award. *artur.izmaylov@utoronto.ca

1 A. Peruzzo, J. McClean, P. Shadbolt, M.-H. Yung, X.-Q. Zhou, P. J. Love, A. Aspuru-Guzik, and J. L. O'Brien, Nat. Commun. 5, 4213 (2014).

2 J. R. McClean, J. Romero, R. Babbush, and A. AspuruGuzik, N. J. Phys. 18, 023023 (2016).

3 D. Wecker, M. B. Hastings, and M. Troyer, Phys. Rev. A 92, 042303 (2015).

4 P. Jordan and E. Wigner, Z. Phys. 47, 631 (1928).

5 A. Aspuru-Guzik, A. D. Dutoi, P. J. Love, and M. HeadGordon, Science 309, 1704 (2005).

6 S. B. Bravyi and A. Y. Kitaev, Ann. Phys. 298, 210 (2002).

7 J. T. Seeley, M. J. Richard, and P. J. Love, J. Chem. Phys. 137, 224109 (2012)

8 A. Tranter, S. Sofia, J. Seeley, M. Kaicher, J. McClean, R. Babbush, P. V. Coveney, F. Mintert, F. Wilhelm, and P. J. Love, Int. J. Quantum Chem. 115, 1431 (2015).

9 K. Setia and J. D. Whitfield, ArXiv e-prints (2017), arXiv:1712.00446 [quant-ph].

10 V. Havlíček, M. Troyer, and J. D. Whitfield, Phys. Rev. A 95, 032332 (2017).

11 A. Kandala, A. Mezzacapo, K. Temme, M. Takita, M. Brink, J. M. Chow, and J. M. Gambetta, Nature 549, 242 (2017).

12 I. G. Ryabinkin, S. N. Genin, and A. F. Izmaylov, J. Chem. Theory Comp. 15, 249 (2019).

13 Y. Nam, J.-S. Chen, N. C. Pisenti, K. Wright, C. Delaney, D. Maslov, K. R. Brown, S. Allen, J. M. Amini, J. Apisdorf, K. M. Beck, A. Blinov, V. Chaplin, M. Chmielewski, C. Collins, S. Debnath, A. M. Ducore, K. M. Hudek, M. Keesan, S. M. Kreikemeier, J. Mizrahi, P. Solomon, M. Williams, J. D. Wong-Campos, C. Monroe, and J. Kim, arXiv.org (2019), 1902.10171v2.

14 G. Harsha, T. Shiozaki, and G. E. Scuseria, J. Chem. Phys. 148, 044107 (2018).

15 B. T. Gard, L. Zhu, G. S. Barron, N. J. Mayhall, S. E. Economou, and E. Barnes, arXiv.org (2019), 1904.10910v1.

16 M. Ganzhorn, D. J. Egger, P. K. Barkoutsos, P. Ollitrault, G. Salis, N. Moll, A. Fuhrer, P. Mueller, S. Woerner, I. Tavernelli, and S. Filipp, arXiv.org (2018), 1809.05057v1.

17 S. Bravyi, J. M. Gambetta, A. Mezzacapo, and K. Temme, ArXiv e-prints (2017), arXiv:1701.08213 [quant-ph].

18 X. Bonet-Monroig, R. Sagastizabal, M. Singh, and T. E. O'Brien, Phys. Rev. A 98, 062339 (2018).

19 S. McArdle, X. Yuan, and S. Benjamin, Phys. Rev. Lett. 122, 180501 (2019).

20 N. Moll, A. Fuhrer, P. Staar, and I. Tavernelli, J. Phys. A: Math. Theor. 49, 295301 (2016).
21 I. G. Ryabinkin and S. N. Genin, arXiv.org (2018), $1812.09812 \mathrm{v} 1$.

${ }^{22}$ V. Verteletskyi, T.-C. Yen, and A. F. Izmaylov, arXiv.org (2019), 1907.03358.

23 A. F. Izmaylov, T.-C. Yen, and I. G. Ryabinkin, Chem. Sci. 10, 3746 (2019).

24 A. F. Izmaylov, T.-C. Yen, R. A. Lang, and V. Verteletskyi, arXiv.org (2019), 1907.09040.

25 T.-C. Yen, V. Verteletskyi, and A. F. Izmaylov, arXiv.org (2019), 1907.09386.

26 Y. Qiu, T. M. Henderson, J. Zhao, and G. E. Scuseria, J. Chem. Phys. 149, 164108 (2018).

27 A. O. Barut and R. Raczka, Theory of Group Representations and Applications (Polish Scientific Publisher, 1980).

28 J. Fuchs and C. Schweigert, Symmetries, Lie Algebras, and Representations (Cambridge University Press, 1997).

29 R. Gilmore, Lie Groups, Physics, and Geometry: An Introduction for Physicists, Engineers and Chemists (Cambridge University Press, 2008).

30 A. F. Izmaylov, J. Phys. Chem. A 123, 3429 (2019).

31 P.-O. Löwdin, Phys. Rev. 97, 1509 (1955).

32 I. M. Gel'fand, Lectures on Linear Algebra (Interscience Publishers, Inc., New York, 1961).

33 T. Helgaker, P. Jorgensen, and J. Olsen, Molecular Electronic-structure Theory (Wiley, 2000).

34 I. G. Ryabinkin, T.-C. Yen, S. N. Genin, and A. F. Izmaylov, J. Chem. Theory Comp. 14, 6317 (2018).

35 I. G. Ryabinkin, S. N. Genin, and A. F. Izmaylov, J. Chem. Phys. 149, 214105 (2018).

\section{APPENDIX A: CONSTRAINING VARIANCE IMPOSES THE EXACT SYMMETRY}

Let us consider variance for wavefunction $|\psi\rangle$, which has an orthogonal complement spanned by the orthonormal functions $\left\{\left|\phi_{k}\right\rangle\right\}$ so that $|\psi\rangle\left\langle\psi\left|+\sum_{k}\right| \phi_{k}\right\rangle\left\langle\phi_{k}\right|=\mathbb{1}$

$$
\begin{aligned}
& \left\langle\psi\left|\hat{O}_{i}^{2}\right| \psi\right\rangle-\left\langle\psi\left|\hat{O}_{i}\right| \psi\right\rangle^{2}=\left\langle\psi\left|\hat{O}_{i} \mathbb{\mathbb { 1 }} \hat{O}_{i}\right| \psi\right\rangle \\
- & \left\langle\psi\left|\hat{O}_{i}\right| \psi\right\rangle\left\langle\psi\left|\hat{O}_{i}\right| \psi\right\rangle \\
= & \sum_{k}\left\langle\psi\left|\hat{O}_{i}\right| \phi_{k}\right\rangle\left\langle\phi_{k}\left|\hat{O}_{i}\right| \psi\right\rangle \\
= & \sum_{k}\left|\left\langle\psi\left|\hat{O}_{i}\right| \phi_{k}\right\rangle\right|^{2} .
\end{aligned}
$$


Action of $\hat{O}_{i}$ on $\langle\psi|$ can always be presented as

$$
\langle\psi| \hat{O}_{i}=\alpha\langle\psi|+\sum_{k} \beta_{k}\left\langle\phi_{k}\right| .
$$

Using this in the variance expression, we can write

$$
\begin{aligned}
\sum_{k}\left|\left\langle\psi\left|\hat{O}_{i}\right| \phi_{k}\right\rangle\right|^{2} & =\sum_{k}\left|\sum_{k^{\prime}} \beta_{k^{\prime}}\left\langle\phi_{k^{\prime}} \mid \phi_{k}\right\rangle\right|^{2} \\
& =\sum_{k}\left|\sum_{k^{\prime}} \beta_{k^{\prime}} \delta_{k k^{\prime}}\right|^{2}=\sum_{k}\left|\beta_{k}\right|^{2}
\end{aligned}
$$

therefore, for the zero variance, all $\beta_{k}$ must be zero, which would make $\langle\psi|$ an eigenstate of $\hat{O}_{i}$ according to Eq. (56).

\section{APPENDIX B: C $_{3 v}$ GROUP PROJECTORS FOR AN E-TYPE ORBITAL BASIS}

To illustrate a non-abelian case for point group projection, we consider construction of the projector for irreducible representations of $C_{3 v}$. Orbitals in a symmetry adapted $C_{3 v}$ basis transforming as $A_{1}$ or $A_{2}$ do not undergo mixing, and hence corresponding operators are similar to the abelian case. We thus concentrate on a doubly degenerate orbital subset transforming as the $E$-type components $\left\{\phi_{x}, \phi_{y}\right\}$, and assume $\phi_{x}$ and $\phi_{y}$ are mapped to even index qubits $i$ and $j$ using the BK transformation. We emphasize that the following operators are valid only within the degenerate $E$ orbital subspace. The size of the unitary operators required in the projector scale by a maximum factor of 2 for every $A_{1}$ or $A_{2}$ adapted orbital added to the basis. We set $\phi_{x}$ and $\phi_{y}$ such that they transform under action of $\hat{C}_{3}$ as

$$
\begin{aligned}
\hat{C}_{3} \phi_{x} & =-\frac{1}{2} \phi_{x}+\frac{\sqrt{3}}{2} \phi_{y}, \\
\hat{C}_{3} \phi_{y} & =-\frac{\sqrt{3}}{2} \phi_{x}-\frac{1}{2} \phi_{y} .
\end{aligned}
$$

The elements of $C_{3 v}$ are $\left\{\mathbb{1}, \hat{C}_{3}, \hat{C}_{3}^{2}, \hat{\sigma}_{v}, \hat{\sigma}_{v}^{\prime}, \hat{\sigma}_{v}^{\prime \prime}\right\}$, thus the form of the projector for an arbitrary irreducible representation $\Gamma$ of $C_{3 v}$ is

$$
\begin{aligned}
\hat{P}_{\Gamma} & =\frac{1}{6}\left(\chi_{\Gamma}^{*}(\mathbb{1}) \mathbb{1}+\chi_{\Gamma}^{*}\left(\hat{C}_{3}\right) \hat{C}_{3}+\chi_{\Gamma}^{*}\left(\hat{C}_{3}^{2}\right) \hat{C}_{3}^{2}\right. \\
& \left.+\chi_{\Gamma}^{*}\left(\hat{\sigma}_{v}\right) \hat{\sigma}_{v}+\chi_{\Gamma}^{*}\left(\hat{\sigma}_{v}^{\prime}\right) \hat{\sigma}_{v}^{\prime}+\chi_{\Gamma}^{*}\left(\hat{\sigma}_{v}^{\prime \prime}\right) \hat{\sigma}_{v}^{\prime \prime}\right) .
\end{aligned}
$$

The setting of $\phi_{x}$ and $\phi_{y}$ in Eqs. (59) and (60) causes transformation matrix $\mathbf{C}_{3}$ to result in $\kappa_{11}=\kappa_{22}=0$, $\kappa_{12}=\frac{2 \pi}{3}$ and $\kappa_{21}=-\kappa_{12}$. The exponentiated sum for $\hat{C}_{3}$ thus takes the form

$$
\begin{aligned}
\hat{C}_{3} & =\exp \left(i \frac{\pi}{3}\left(\hat{x}_{i} \hat{x}_{j}+\hat{y}_{i} \hat{y}_{j}\right) \hat{x}_{U_{i j} / \alpha_{i j}} \hat{z}_{P_{i j} / \alpha_{i j}} \hat{y}_{\alpha_{i j}}\right) \\
& =\frac{1}{4} \mathbb{1}+\frac{3}{4} \hat{z}_{i} \hat{z}_{j}+i \frac{\sqrt{3}}{4}\left(\hat{y}_{i} \hat{y}_{j}+\hat{x}_{i} \hat{x}_{j}\right) \hat{x}_{U_{i j} / \alpha_{i j}} \hat{z}_{P_{i j} / \alpha_{i j}} \hat{y}_{\alpha_{i j}},
\end{aligned}
$$

where

$$
\begin{aligned}
U_{i j} & \equiv U(i) \triangle U(j), \\
P_{i j} & \equiv P(i) \triangle P(j), \\
\alpha_{i j} & \equiv U(i) \cap P(j) .
\end{aligned}
$$

The symbol $\triangle$ denotes the disjoint union, and $U(i)$ and $P(i)$ respectively denote the update set and parity set of $i$.

$\hat{C}_{3}^{2}$ is analogous to $\hat{C}_{3}$ case but with a sign swapping between $\kappa_{12}$ and $\kappa_{21}$, resulting in

$\hat{C}_{3}^{2}=\frac{1}{4} \mathbb{1}+\frac{3}{4} \hat{z}_{i} \hat{z}_{j}-i \frac{\sqrt{3}}{4}\left(\hat{y}_{i} \hat{y}_{j}+\hat{x}_{i} \hat{x}_{j}\right) \hat{x}_{U_{i j} / \alpha_{i j}} \hat{z}_{P_{i j} / \alpha_{i j}} \hat{y}_{\alpha_{i j}}$

Setting $\hat{\sigma}_{v}$ as the reflection plane defined by the $x$ and $z$ axis, then $\hat{\sigma}_{v} \phi_{x}=\phi_{x}$ and $\hat{\sigma}_{v} \phi_{y}=-\phi_{y}$. The only nonzero element in $\kappa$ is $\kappa_{22}=i \pi$. It follows that $\hat{\sigma}_{v}$ takes a simple abelian form

$$
\hat{\sigma}_{v}=\hat{z}_{\underline{F(j)}}=\hat{z}_{j},
$$

where the second equality holds since $j$ was chosen to be even. Setting $\hat{\sigma}_{v}^{\prime}$ as the reflection plane acting on the $E$ components as

$$
\begin{aligned}
& \hat{\sigma}_{v}^{\prime} \phi_{x}=-\frac{1}{2} \phi_{x}+\frac{\sqrt{3}}{2} \phi_{y} \\
& \hat{\sigma}_{v}^{\prime} \phi_{y}=\frac{\sqrt{3}}{2} \phi_{x}+\frac{1}{2} \phi_{y},
\end{aligned}
$$

gives elements $\kappa_{11}=i 3 \pi / 4, \kappa_{22}=i \pi / 4$, and $\kappa_{12}=\kappa_{21}=$ $-i \sqrt{3} / 4$. The resulting qubit space unitary operator is

$$
\begin{aligned}
\hat{\sigma}_{v}= & \exp \left(\frac { i \pi } { 8 } \left(3 \hat{z}_{i}+\hat{z}_{j}-4 \mathbb{1}\right.\right. \\
& \left.-\sqrt{3}\left(\hat{x}_{i} \hat{y}_{j}-\hat{y}_{i} \hat{x}_{j}\right) \hat{x}_{U_{i j} / \alpha_{i j}} \hat{z}_{P_{i j} / \alpha_{i j}} \hat{y}_{\alpha_{i j}}\right) \\
= & \frac{3}{4} \hat{z}_{i}+\frac{1}{4} \hat{z}_{j}-\frac{\sqrt{3}}{4}\left(\hat{x}_{i} \hat{y}_{j}-\hat{y}_{i} \hat{x}_{j}\right) \hat{x}_{U_{i j} / \alpha_{i j}} \hat{z}_{P_{i j} / \alpha_{i j}} \hat{y}_{\alpha_{i j}} .
\end{aligned}
$$

The remaining reflection plane $\hat{\sigma}_{v}^{\prime \prime}$ must act on the $E$ components as

$$
\begin{aligned}
\hat{\sigma}_{v}^{\prime \prime} \phi_{x} & =-\frac{1}{2} \phi_{x}-\frac{\sqrt{3}}{2} \phi_{y}, \\
\hat{\sigma}_{v}^{\prime \prime} \phi_{y} & =-\frac{\sqrt{3}}{2} \phi_{x}+\frac{1}{2} \phi_{x} \phi_{y},
\end{aligned}
$$

leading to same case as $\hat{\sigma}_{v}^{\prime}$ but with positive $\kappa_{12}$ and $\kappa_{21}$, giving qubit unitary form:

$$
\begin{aligned}
\hat{\sigma}_{v}^{\prime \prime} & =\frac{3}{4} \hat{z}_{i}+\frac{1}{4} \hat{z}_{i} \\
& +\frac{\sqrt{3}}{4}\left(\hat{x}_{i} \hat{y}_{j}-\hat{y}_{i} \hat{x}_{j}\right) \hat{x}_{U_{i j} / \alpha_{i j}} \hat{z}_{P_{i j} / \alpha_{i j}} \hat{y}_{\alpha_{i j}} .
\end{aligned}
$$

Further simplification may arise by classes present within the point group of interest. The two non-trivial classes in 
$C_{3 v}$ are $\left\{\hat{C}_{3}, \hat{C}_{3}^{2}\right\}$ and $\left\{\hat{\sigma}_{v}, \hat{\sigma}_{v}^{\prime}, \hat{\sigma}_{v}^{\prime \prime}\right\}$. Since elements within a class will have identical characters for a given irreducible representation, unitaries in the projector expression may be factored leading to $n$ groupings of unitary operations for $n$ classes within the group, leading to favorable cancellation of multi-qubit operations in Eq.(61).
The resultant $C_{3 v}$ projector is

$$
\hat{P}_{\Gamma}=\frac{d_{\Gamma}}{6}\left(\chi_{\Gamma}^{*}(\mathbb{1}) \mathbb{1}+\frac{\chi_{\Gamma}^{*}\left(\hat{C}_{3}\right)}{2}\left(\mathbb{1}+3 \hat{z}_{i} \hat{z}_{j}\right)+\frac{3 \chi_{\Gamma}^{*}\left(\hat{\sigma}_{v}\right)}{2}\left(\hat{z}_{i}+\hat{z}_{j}\right)\right)
$$

